\title{
Collider signatures of gravitino dark matter with a sneutrino NLSP
}

\author{
Laura Covi $^{1}$, Sabine $\mathrm{Kraml}^{2}$ \\ ${ }^{1}$ Deutsches Elektronen-Synchrotron DESY, D-22603 Hamburg, Germany \\ ${ }^{2}$ CERN, CH-1211 Geneva 23, Switzerland
}

\begin{abstract}
For gravitino dark matter with conserved R-parity and mass in the $\mathrm{GeV}$ range, very strong constraints from Big Bang Nucleosynthesis exclude the popular NLSP candidates like neutralino and charged sleptons. In this letter we therefore draw attention to the case of a sneutrino NLSP, that is naturally realised in the context of gaugino mediation. We find interesting collider signatures, characterised by soft jets or leptons due to the small sneutrino-stau mass splitting. Moreover, the lightest neutralino can have visible decays into staus, and in some part of the parameter space also into selectrons and smuons. We also show the importance of coannihilation effects for the evaluation of the BBN constraints.
\end{abstract}

\section{Introduction}

If the gravitino is the lightest supersymmetric particle (LSP) and stable (with conserved Rparity) or sufficiently long-lived, it is a good candidate for the Cold Dark Matter (CDM). At high temperatures, gravitinos are produced by thermal scatterings even if they are not in thermal equilibrium. The resulting energy density is approximately given by $[1,2]$

$$
\Omega_{3 / 2}^{\mathrm{th}} h^{2} \simeq 0.27\left(\frac{T_{\mathrm{R}}}{10^{10} \mathrm{GeV}}\right)\left(\frac{100 \mathrm{GeV}}{m_{3 / 2}}\right)\left(\frac{m_{\tilde{g}}}{1 \mathrm{TeV}}\right)^{2}
$$

where $m_{\tilde{g}}$ is the running gluino mass evaluated at low energy. For a given $m_{\tilde{g}}$, the maximal possible reheating temperature $T_{\mathrm{R}}$ is obtained for the heaviest allowed gravitino mass.

Gravitinos are also produced non-thermally via the decays of the next-to-lightest supersymmetric particle (NLSP), leading to

$$
\Omega_{3 / 2}^{\text {non-th }} h^{2}=\frac{m_{3 / 2}}{m_{\mathrm{NLSP}}} \Omega_{\mathrm{NLSP}}^{\mathrm{th}} h^{2}
$$

Here $\Omega_{\mathrm{NLSP}}^{\text {th }} h^{2}$ is the would-be relic density of the NLSP from thermal freeze-out if it did not decay. The total energy density of the gravitino LSP, $\Omega_{3 / 2} h^{2}=\Omega_{3 / 2}^{\text {th }} h^{2}+\Omega_{3 / 2}^{\text {non-th }} h^{2}$, has to be equal or smaller than the cosmologically observed CDM density. In particular, if gravitinos should make up all the cold dark matter, $0.094 \leq \Omega_{3 / 2} h^{2} \leq 0.135$ [3]. In general the right CDM 
abundance can be obtained from both mechanisms for supersymmetric masses in the GeV-TeV region $[1,4]$.

On the other hand, Big Bang Nucleosynthesis (BBN) severely constrains the nature, the lifetime and the freeze-out abundance of the NLSP. This is because the electromagnetic and hadronic energy released by the NLSP decays into the gravitino at comparatively late times $(t>100 \mathrm{~s})$ can alter the primordial abundances of light elements [5,6]. Moreover if the NLSP is charged, also bound state effects can change heavily the rates of the nuclear reactions and modify the BBN predictions [7-9].

In fact, most NLSPs are incompatible with BBN, as long as their lifetime is not shorter than $10^{3}$ s, i.e. the supersymmetric spectrum is very heavy, or their abundance is not strongly suppressed compared to that expected by thermal freeze-out, e.g. diluted by late entropy production $[10,11]$. So in the minimal setting of simple freeze-out and masses for both gravitino and NLSP in the GeV range, neutralino [12-16] and stau [6,7,17] NLSP are incompatible with BBN 1 For completeness, let us mention that a stop NLSP could be viable in some particular region of the supersymmetric parameter space [18]. A sneutrino NLSP, on the other hand, is neutral and decays mainly into gravitino and neutrino, which are not electromagnetically or hadronically active. The BBN bounds $[19,20]$ arising from the neutrino interactions and the subdominant decay channel into quarks are much weaker than those for a neutralino or charged slepton NLSP. In this study, we therefore consider a sneutrino NLSP as an interesting alternative.

The paper is organised as follows. In Section 2 we briefly explain the model of gaugino mediation. In Section 3 we discuss the sparticle spectrum in this model, focusing in particular on the parameter range which leads to a sneutrino NLSP. In Section 4 we evaluate the BBN constraints on the sneutrino NLSP scenario, going beyond the approximation used in [20]. In Section 5 we discuss the signatures at LHC and ILC, and Section 6 finally contains our conclusions.

\section{The model}

In general, in models of supersymmetry (SUSY) breaking with universal scalar and gaugino masses, the right-chiral charged sleptons are lighter than the left-chiral ones and the sneutrinos. The reason is that the running of $m_{\tilde{l}_{R}}^{2}$ is dominated by $U(1)_{Y}$ D-term contributions, while $m_{\tilde{l}_{L}}^{2}$ receives $S U(2)_{L}$ and $U(1)_{Y}$ D-term corrections. This picture changes, however, for nonuniversal SUSY breaking parameters at the high scale, especially for non-universal Higgs-mass parameters with $m_{H_{1}}^{2}-m_{H_{2}}^{2}>0$, see e.g. [21].

A particularly attractive realisation of non-universal boundary conditions is the case of gaugino mediation [22,23], where supersymmetry breaking occurs on a four-dimensional brane within a higher-dimensional theory. In such a setting, fields which live in different places will naturally feel such breaking with different strength. Gauge and Higgs superfields living in the bulk couple directly to the chiral superfield $S$ responsible for SUSY breaking, which is localised on one of the four-dimensional branes. The gaugino and Higgs fields hence acquire soft SUSYbreaking masses at tree level. Squarks and sleptons, on the other hand, are confined to some other branes, without direct coupling to $S$ and this yields no-scale boundary conditions [24,25]

\footnotetext{
${ }^{1}$ Of course most of the constraints are weakened or disappear for shorter NLSP lifetime, i.e. lighter gravitino masses or larger NLSP masses. We recall that the NLSP lifetime is given approximately by $\tau_{\mathrm{NLSP}} \simeq$ $10^{6} \mathrm{~s}\left(\frac{m_{3 / 2}}{10 \mathrm{GeV}}\right)^{2}\left(\frac{m_{\mathrm{NLSP}}}{100 \mathrm{GeV}}\right)^{-5}$.
} 
for their masses. We therefore have the following boundary conditions at the compactification scale $M_{C}$ [23]:

$$
\begin{aligned}
& g_{1}=g_{2}=g_{3}=g \simeq 1 / \sqrt{2}, \\
& M_{1}=M_{2}=M_{3}=m_{1 / 2}, \\
& m_{0}^{2}=0 \text { for all squarks and sleptons, } \\
& A_{0}=0 \\
& \mu, B \mu, m_{H_{1,2}}^{2} \neq 0,
\end{aligned}
$$

with GUT charge normalisation used for $g_{1}$. The superparticle spectrum is determined from these boundary conditions and the renormalisation group equations. The free parameters of the model are hence $m_{1 / 2}, m_{H_{1}}^{2}, m_{H_{2}}^{2}, \tan \beta$, and the sign of $\mu ;|\mu|$ being determined by radiative electroweak symmetry breaking.

The model favours moderate values of $\tan \beta$ between about 10 and 25 . The parameter ranges leading to a viable low-energy spectrum were discussed in [26, 27], assuming $M_{C}=$ $M_{\mathrm{GUT}}$. In [28] it was shown that either the lightest neutralino or the gravitino can be viable dark matter candidates in this model. In particular, Ref. [28] discussed the possibility of a gravitino LSP with a (tau-)sneutrino NLSP for $m_{1 / 2}=500 \mathrm{GeV}$ and $\tan \beta=10$ and 20 . In this case, the sneutrino NLSP occurs for $m_{H_{2}}^{2} \lesssim 0.5 \mathrm{TeV}^{2}$ and large values of $m_{H_{1}}^{2}$ of roughly $2-3 \mathrm{TeV}^{2}$. Ref. [27] also discussed the collider phenomenology of gaugino mediation, concentrating however on the case of a neutralino LSP.

\section{Sparticle spectrum in gaugino mediation with a sneutrino NLSP}

We here investigate the SUSY spectrum in the gaugino-mediation model in more detail. We assume that the gravitino is the LSP and concentrate on scenarios with a sneutrino NLSP. Following [26, 28], we take $m_{t}=172.5 \mathrm{GeV}, m_{b}\left(m_{b}\right)=4.25 \mathrm{GeV}$ and $\alpha_{s}^{\mathrm{SM} \overline{\mathrm{MS}}}\left(M_{Z}\right)=0.1187$ as SM input parameters, and consider $m_{3 / 2}=10 \mathrm{GeV}$ as lower bound for the gravitino mass (the upper bound being given by the NLSP mass and the BBN constraints). Moreover, we take $M_{C}=M_{\mathrm{GUT}}$. We use SOFTSUSY 2.0 .10 [29] to compute the sparticle and Higgs masses and mixing angles, and micrOMEGAs 2.0 [30-32] to compute the primordial abundance of the NLSP.

Figure 1 shows the sneutrino NLSP region in the $m_{H_{1}}^{2}$ versus $m_{1 / 2}$ plane for $\tan \beta=10$ and two values of $m_{H_{2}}^{2}, m_{H_{2}}^{2}=0$ and $0.4 \mathrm{TeV}^{2}$. Also shown are contours of constant $m_{\tilde{\tau}_{1}}-m_{\tilde{\nu}_{\tau}}$ in GeV: since $m_{\tilde{\tau}_{L}}$ and $m_{\tilde{\nu}_{\tau}}$ are driven by the same SUSY-breaking parameter $M_{\tilde{L}_{3}}$, the mass difference between the $\tilde{\nu}_{\tau}$ and the $\tilde{\tau}_{1}$ is always small. The mass of the $\tilde{\nu}_{\tau}$ NLSP goes up to about 250 (230) $\mathrm{GeV}$ for $m_{H_{2}}^{2}=0\left(0.4 \mathrm{TeV}^{2}\right)$ and $m_{1 / 2}=600 \mathrm{GeV}$ in Fig. 1, Comparing with Fig. 4 of [20], one might conclude that the $\tilde{\nu}_{\tau}$ NLSP region of Fig. 1 is in good agreement with BBN; this is discussed in more detail in the next section. For fixed $m_{1 / 2}, m_{\tilde{\nu}_{\tau}}$ decreases with increasing $m_{H_{1}}^{2}$, and so do $m_{\tilde{\tau}_{1}}$ and $m_{\tilde{e}_{L}} \simeq m_{\tilde{\mu}_{L}}$, while $m_{\tilde{\chi}_{1}^{0}}$ remains constant. One therefore finds the mass orderings 2 2 $m_{\tilde{\nu}_{\tau}}<m_{\tilde{\chi}_{1}^{0}}<m_{\tilde{\tau}_{1}}<m_{\tilde{e}_{L}}, m_{\tilde{\nu}_{\tau}}<m_{\tilde{\tau}_{1}}<m_{\tilde{\chi}_{1}^{0}}<m_{\tilde{e}_{L}}$ and $m_{\tilde{\nu}_{\tau}}<m_{\tilde{\tau}_{1}}<m_{\tilde{e}_{L}}<$ $m_{\tilde{\chi}_{1}^{0}}$ within the sneutrino NLSP region. These are labelled A, B, and C, respectively, in Fig. 1 ,

The case of $\tan \beta=20$ is shown in Fig. 2 for $m_{H_{2}}^{2}=0.2$ and $0.4 \mathrm{TeV}^{2}$. Analogous arguments as above apply. Note, however, that here the $\tilde{e}_{L}$ does not become lighter than the $\tilde{\chi}_{1}^{0}$.

\footnotetext{
${ }^{2}$ Since selectrons and smuons are practically degenerate, in the following $\tilde{e}$ implicitly means selectrons and smuons.
} 

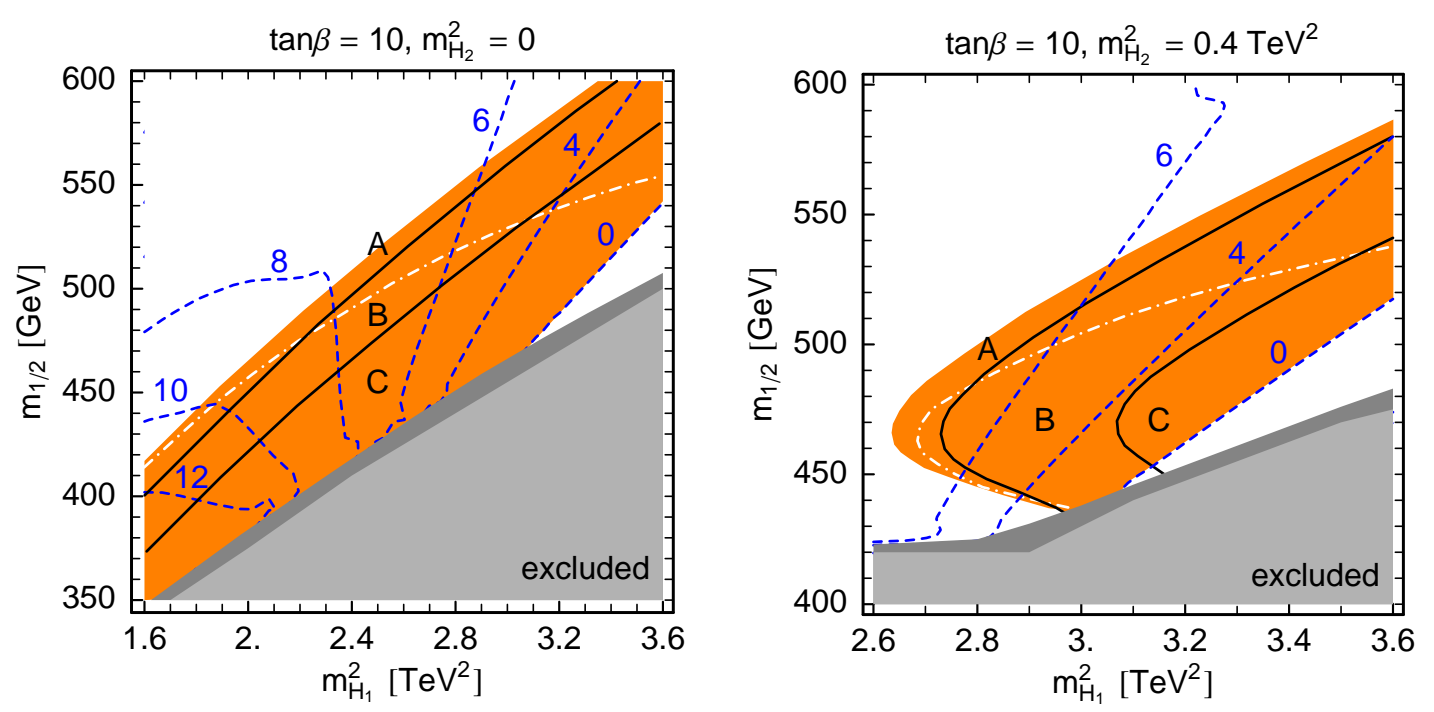

Fig. 1: Sneutrino NLSP regions (in orange) in the $m_{H_{1}}^{2}$ versus $m_{1 / 2}$ plane for $\tan \beta=10$ and $m_{H_{2}}^{2}=0$ (left) and $m_{H_{2}}^{2}=0.4 \mathrm{TeV}^{2}$ (right). The blue dashed lines show contours of constant $m_{\tilde{\tau}_{1}}-m_{\tilde{\nu}_{\tau}}$ in GeV. The full black lines separate subregions of different mass ordering: $m_{\tilde{\nu}_{\tau}}<m_{\tilde{\chi}_{1}^{0}}<m_{\tilde{\tau}_{1}}<m_{\tilde{e}_{L}}$ in A, $m_{\tilde{\nu}_{\tau}}<m_{\tilde{\tau}_{1}}<m_{\tilde{\chi}_{1}^{0}}<m_{\tilde{e}_{L}}$ in $\mathrm{B}$, and $m_{\tilde{\nu}_{\tau}}<m_{\tilde{\tau}_{1}}<m_{\tilde{e}_{L}}<m_{\tilde{\chi}_{1}^{0}}$ in C. Below the white dash-dotted line, the BBN bounds are satisfied for any gravitino mass, i.e. $m_{\tilde{\nu}} Y_{\tilde{\nu}} \leq 3 \times 10^{-11} \mathrm{GeV}$, as discussed in the text. In the light grey regions, no viable spectrum is obtained, while in the narrow medium grey strips, $m_{\tilde{\tau}_{1}}<90 \mathrm{GeV}$.

Moreover, the $\tilde{\nu}_{\tau}-\tilde{\tau}_{1}$ mass difference shows a different behaviour as compared to $\tan \beta=10$ : At $\tan \beta=10$ and small $m_{H_{1}}^{2}, m_{\tilde{\nu}_{\tau}}<m_{\tilde{\tau}_{1}}$ with the mass difference becoming smaller as $m_{H_{1}}^{2}$ increases. At $\tan \beta=20$, the $\tilde{\tau}_{1}$ is first lighter than the $\tilde{\nu}_{\tau}$; with increasing $m_{H_{1}}^{2}, m_{\tilde{\nu}_{\tau}}$ decreases faster than $m_{\tilde{\tau}_{1}}$, eventually leading to $m_{\tilde{\nu}_{\tau}}<m_{\tilde{\tau}_{1}}$. This is why the contour of $m_{\tilde{\tau}_{1}}-m_{\tilde{\nu}_{\tau}}=0$ is on the upper-left edge of the $\tilde{\nu}_{\tau}$ NLSP region in Fig. 2, while it is on the lower-right edge in Fig. 1.

A comment is in order concerning the LEP limit on the light Higgs mass. Demanding $m_{h^{0}} \geq 114.5 \mathrm{GeV}$ would constrain $m_{1 / 2}$ to $m_{1 / 2} \gtrsim 500$ (440) GeV in Fig. 11 (2). However, there is still a 2-3 GeV uncertainty in the evaluation of $m_{h^{0}}$. If this is taken into account, the full parameter range considered is allowed.

\section{Sneutrino abundance and BBN constraints}

Even if the sneutrino is neutral and decays mainly into weakly interacting particles, still BBN constraints arise from the subleading decay channels. According to [20], Figure 4, such bounds are satisfied for light sneutrinos with masses below $300 \mathrm{GeV}$, because the branching ratios into quarks via virtual $Z, W$ are rather small. This conclusion was obtained through an estimate of the sneutrino freeze-out abundance of

$$
Y_{\tilde{\nu}} \simeq 2 \times 10^{-14}\left(\frac{m_{\tilde{\nu}}}{100 \mathrm{GeV}}\right)
$$

In our case though, due to the close spacing between the different masses, co-annihilation effects [21] become important, making this estimate unreliable. Here note that co-annihilation effects can both decrease or increase the particle yield. The latter can occur if the co-annihilation cross section is small, due to the presence in the thermal bath of the slightly heavier states that can decay into the NLSP [33]. We therefore use micrOMEGAs 2 . 0 [30-32] to compute $Y_{\tilde{\nu}}$ 

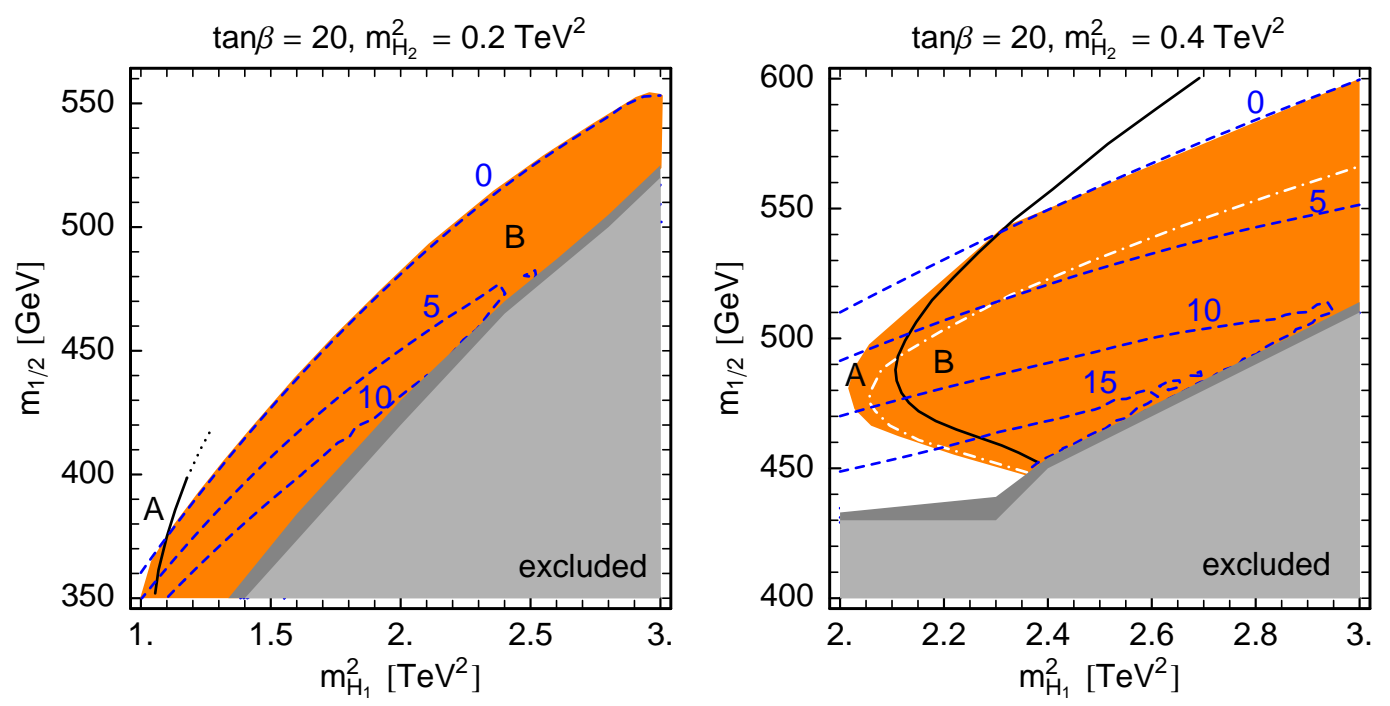

Fig. 2: Same as Fig. 11 but for $\tan \beta=20$ and $m_{H_{2}}^{2}=0.2 \mathrm{TeV}^{2}$ (left) and $m_{H_{2}}^{2}=0.4 \mathrm{TeV}^{2}$ (right). BBN bounds play no role in the left-hand panel.

numerically without approximation, and obtain that in our region of the parameter space the sneutrino abundance

$$
m_{\tilde{\nu}} Y_{\tilde{\nu}}=3.63 \times 10^{-9} \mathrm{GeV} \Omega_{\tilde{\nu}}^{\text {th }} h^{2}
$$

can be as large as $10^{-10} \mathrm{GeV}$. This value violates the general bounds given in [20] for a gravitino mass in the range $2-50 \mathrm{GeV}$. The limit for a gravitino with a mass of about $10 \mathrm{GeV}$ is in fact $m_{\tilde{\nu}} Y_{\tilde{\nu}}<3 \times 10^{-11} \mathrm{GeV}$, which is shown as dash-dotted line in Figs. 1 and 2, For a gravitino mass of $50 \mathrm{GeV}$ or larger, or for a sneutrino decay branching ratio into hadrons substantially smaller than $10^{-3}$, this BBN bound becomes much weaker and disappears in our parameter region. We will consider in the following benchmark points where the BBN constraints are satisfied.

Last but not least, since $\Omega_{\mathrm{NLSP}}^{\mathrm{th}} h^{2}$ is very small, typically $\mathcal{O}\left(10^{-3}\right)$, throughout the $\tilde{\nu}_{\tau}$ NLSP region, $\Omega_{3 / 2}^{\text {non-th }} h^{2}$ is negligible and almost all the gravitino dark matter has to be produced thermally. Requiring $\Omega_{3 / 2} h^{2} \simeq 0.1$ leads to $T_{R} \sim 10^{8}-10^{9} \mathrm{GeV}$ for $m_{\tilde{g}} \sim 1 \mathrm{TeV}$ and $m_{\widetilde{G}}$ in the range of $10-100 \mathrm{GeV}$.

\section{Collider signatures}

The collider signatures are characterised by the small $\tilde{\nu}_{\tau}-\tilde{\tau}_{1}$ mass difference. As mentioned, we can have the cases $m_{\tilde{\tau}_{1}}>m_{\tilde{\chi}_{1}^{0}}>m_{\tilde{\nu}_{\tau}}$ (region A) or $m_{\tilde{\chi}_{1}^{0}}>m_{\tilde{\tau}_{1}}>m_{\tilde{\nu}_{\tau}}$ (region B). In the former the $\tilde{\chi}_{1}^{0}$ decays via $\tilde{\chi}_{1}^{0} \rightarrow \nu \tilde{\nu}_{\tau}$, while in the latter it can also decay directly into the visible channel $\tilde{\chi}_{1}^{0} \rightarrow \tau \tilde{\tau}_{1}$. If also the $\tilde{e}_{L}$ is lighter than the $\tilde{\chi}_{1}^{0}$ (region $\mathrm{C}$ ), $\tilde{\chi}_{1}^{0} \rightarrow e^{ \pm} \tilde{e}_{L}^{\mp}$ is possible in addition. The NLSP decay into the gravitino, $\tilde{\nu}_{\tau} \rightarrow \nu \widetilde{G}$, is of course invisible, regardless of the $\tilde{\nu}_{\tau}$ lifetime. On the other hand, even if such a decay is impossible to detect, it is clear that the sneutrino cannot be stable and the dominant DM component, since it has been already excluded by direct searches [34].

The $\tilde{\tau}_{1}$ can decay into $\tau \tilde{\chi}_{1}^{0}$ if $m_{\tilde{\tau}_{1}}>m_{\tilde{\chi}_{1}^{0}}+m_{\tau}$; its 2-body decays into the NLSP, $\tilde{\tau}_{1}^{ \pm} \rightarrow$ $W^{ \pm} \tilde{\nu}_{\tau}$ or $H^{ \pm} \tilde{\nu}_{\tau}$, are however kinematically forbidden due to the small mass splittings. For 


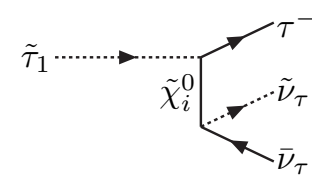

(a)

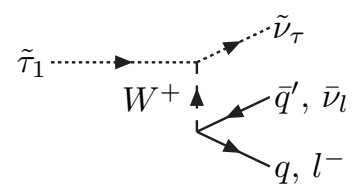

(d)

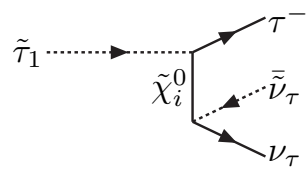

(b)

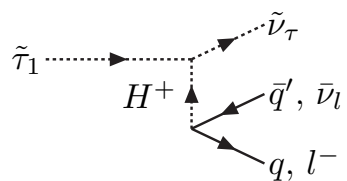

(e)

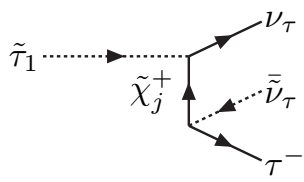

(c)

Fig. 3: Feynman diagrams for stau three-body decays into a sneutrino LSP $(i=1 \ldots 4, j=1,2)$. The dominant contribution comes from the $W$ exchange of diagram (d).

$m_{\tilde{\tau}_{1}}<m_{\tilde{\chi}_{1}^{0}}+m_{\tau}$, the $\tilde{\tau}_{1}$ hence only has 3-body decays leading to $f \bar{f}^{\prime}$ plus missing energy as shown in Fig. 3. The dominant contribution comes from the diagram with the virtual $W$ boson. The resulting $\tilde{\tau}_{1}$ lifetime in this channel is approximately given by

$$
\Gamma_{\tilde{\tau}}^{-1} \simeq \frac{2(2 \pi)^{3}}{3 G_{F}^{2} m_{\tilde{\tau}}^{5}} F^{-1}\left(\frac{m_{\tilde{\nu}}^{2}}{m_{\tilde{\tau}}^{2}}\right)=0.8 \times 10^{-16} \mathrm{~s}\left(\frac{m_{\tilde{\tau}}}{100 \mathrm{GeV}}\right)^{-5}\left(\frac{F\left(m_{\tilde{\nu}}^{2} / m_{\tilde{\tau}}^{2}\right)}{F(0.9)}\right)^{-1}
$$

where, after neglecting the $W$ momentum and the SM particle masses, we have

$$
F(a)=\int_{2 \sqrt{a}}^{1+a} d x\left(x^{2}-4 a\right)^{3 / 2}
$$

So for $m_{\tilde{\tau}_{1}}-m_{\tilde{\nu}_{\tau}} \sim 5-10 \mathrm{GeV}$ the lifetime is of the order of $10^{-16}-10^{-18} \mathrm{~s}$; a displaced vertex is only obtained if the $\tilde{\nu}_{\tau}$ and the $\tilde{\tau}_{1}$ are quasi-degenerate.

In the parameter range we consider, squarks and gluinos have masses of about $1 \mathrm{TeV}$, leading to large SUSY cross sections at the LHC. Since $m_{0}=0$, the gluino is always the heaviest sparticle and decays into $q \tilde{q}$. Moreover, $m_{\tilde{e}_{L}}<m_{\tilde{e}_{R}}$ and the left-chiral sleptons can be light enough to be produced in cascade decays 3 In the following, we discuss these cascade decays in more detail. If the $\tilde{\chi}_{1}^{0}$ is mainly a bino (which is the case for zero or small $m_{H_{2}}^{2}$ ), right-chiral squarks dominantly decay into $q \tilde{\chi}_{1}^{0}$. If $m_{\tilde{\tau}_{1}}+m_{\tau}>m_{\tilde{\chi}_{1}^{0}}>m_{\tilde{\nu}_{\tau}}$, this looks just like the neutralino-LSP case. If, however, $m_{\tilde{\chi}_{1}^{0}}>m_{\tilde{\tau}_{1}}+m_{\tau}>m_{\tilde{\nu}_{\tau}}$, then the $\tilde{\chi}_{1}^{0}$ can decay further into $\tilde{\chi}_{1}^{0} \rightarrow \tau^{ \pm} \tilde{\tau}_{1}^{\mp} \rightarrow \tau^{ \pm} f \bar{f}^{\prime} \tilde{\nu}_{\tau}$. Here note that the $f \bar{f}^{\prime}=\left(q \bar{q}^{\prime}, l \nu_{l}\right)$ will be quite soft. The left-chiral squarks can have more complicated cascade decays. If $m_{\tilde{\chi}_{1}^{0}} \gtrsim m_{\tilde{\tau}_{1}}$, these are generically given by the conventional cascade decays into the $\tilde{\chi}_{1}^{0}$ as in the CMSSM, partly supplemented by $\tilde{\chi}_{1}^{0} \rightarrow \tau^{ \pm} \tilde{\tau}_{1}^{\mp} \rightarrow \tau^{ \pm} f \bar{f}^{\prime} \tilde{\nu}_{\tau}$. The resulting signatures are missing energy plus jets plus (single or di-) leptons PLUS an additional tau, plus additional soft leptons or jets if they can be detected. Examples for such cascades are depicted in Fig. 4. The benchmark point no. 2 of [26] with $m_{1 / 2}=500 \mathrm{GeV}, \tan \beta=10, m_{H_{1}}^{2}=2.7 \mathrm{TeV}^{2}, m_{H_{2}}^{2}=0$ is an illustrative case. The mass spectrum and the most important branching ratios for this point

\footnotetext{
${ }^{3}$ This is in sharp contrast to the CMSSM/mSUGRA case, where $m_{\tilde{e}_{L}}>m_{\tilde{e}_{R}}$, and typically only the right sleptons appear in the cascades.
} 
Table 1: Spectrum and branching ratios for $m_{1 / 2}=500 \mathrm{GeV}, \tan \beta=10, m_{H_{1}}^{2}=2.7 \mathrm{TeV}^{2}, m_{H_{2}}^{2}=0$. As the first and second generation sfermions are practically degenerate, only the first generation is given.

\begin{tabular}{c|r|lll}
\hline Sparticle & Mass [GeV] & Dominant decay modes & \\
\hline$\tilde{g}$ & 1151.8 & $\tilde{q}_{L} q(15 \%), \quad \tilde{q}_{R} q(37 \%), \quad b_{1,2}(19 \%)$, & $\tilde{t}_{1} t(29 \%)$ \\
$\tilde{u}_{L}, \tilde{d}_{L}$ & $1054.0,1062.0$ & $\tilde{\chi}_{2}^{0} q(32 \%), \quad \tilde{\chi}_{1}^{ \pm} q^{\prime}(\sim 60 \%)$ & \\
$\tilde{u}_{R}, \tilde{d}_{R}$ & $971.8,1029.2$ & $\tilde{\chi}_{1}^{0} q(99 \%)$ & \\
$\tilde{t}_{1}$ & 766.3 & $\tilde{\chi}_{1}^{0} t(30 \%), \quad \tilde{\chi}_{1}^{+} b(33 \%)$ & \\
$\tilde{\chi}_{4}^{0}$ & 617.9 & $\tilde{\chi}_{1}^{ \pm} W^{\mp}(46 \%), \quad \tilde{\chi}_{2}^{0} h(19 \%)$ & \\
$\tilde{\chi}_{2}^{ \pm}$ & 614.6 & $\tilde{\chi}_{2}^{0} W^{ \pm}(26 \%), \quad \tilde{\chi}_{1}^{ \pm} Z(22 \%)$ \\
$\tilde{\chi}_{3}^{0}$ & 604.8 & $\tilde{\chi}_{1}^{ \pm} W^{\mp}(56 \%), \quad \tilde{\chi}_{2}^{0} Z(26 \%)$ & \\
$\tilde{e}_{R}$ & 418.3 & $\tilde{\chi}_{1}^{0} e(100 \%)$ & \\
$\tilde{\tau}_{2}$ & 398.8 & $\tilde{\chi}_{1}^{0} \tau(82 \%)$ & \\
$\tilde{\chi}_{1}^{ \pm}$ & 387.4 & $\tilde{e}_{L}^{ \pm} \nu_{e}(15 \%), \quad \tilde{\nu}_{e} e^{ \pm}(17 \%), \quad \tilde{\tau}_{1}^{ \pm} \nu_{\tau}(18 \%), \quad \tilde{\nu}_{\tau} \tau^{ \pm}(19 \%)$ \\
$\tilde{\chi}_{2}^{0}$ & 381.3 & $\tilde{\tau}_{1}^{ \pm} \tau^{\mp}(19 \%), \quad \tilde{e}_{L}^{ \pm} e^{\mp}(16 \%), \quad \tilde{\nu}_{e} \nu_{e}(15 \%)$ \\
$\tilde{e}_{L}$ & 206.5 & $\tilde{\chi}_{1}^{0} e(100 \%)$ & \\
$\tilde{\chi}_{1}^{0}$ & 203.4 & $\tilde{\tau}_{1}^{ \pm} \tau^{\mp}(33 \%), \quad \tilde{\nu}_{\tau} \nu_{\tau}(62 \%)$ & \\
$\tilde{\nu}_{e}$ & 198.5 & $\tilde{\nu}_{\tau} \nu_{e} \bar{\nu}_{\tau}(94 \%)$ & \\
$\tilde{\tau}_{1}$ & 182.3 & $\tilde{\nu}_{\tau} l \nu(32 \%), \quad \tilde{\nu}_{\tau} q \bar{q}^{\prime}(68 \%), \quad \Gamma=2 \times 10^{-8} \mathrm{GeV}$ \\
$\tilde{\nu}_{\tau}$ & 176.1 & $\widetilde{G}_{\tau}, \quad \Omega_{\tilde{\nu}}^{\text {th }} h^{2}=7.2 \times 10^{-3}$ & \\
\hline
\end{tabular}

are given in Table 1. The 2-body decays were computed with SDECAY [35], and the 3-body decay with CALCHEP [36]. The resulting ratios for the decay chains of Fig. 4 are (a) 33\%, (b) $6 \%$, (c) $6.4 \%$, (d) $3.3 \%$, (e) $7 \%$. The sparticle masses can be determined from these cascades through the standard method of invariant-mass distributions of the SM decay products [37-41]; see also $[42,43]$ and references therein. The correct interpretation of the scenario is, however, more involved than in the conventional CMSSM case, and care is needed in order not to falsely conclude to have found SUSY with a neutralino LSP. Notice also that the chain (e) as well as the $\tilde{\tau}_{1} \rightarrow W^{*} \tilde{\nu}_{\tau}$ decays may fake lepton number violation.

So far we have assumed $m_{\tilde{\chi}_{2}^{0}}>m_{\tilde{l}_{L}}>m_{\tilde{\chi}_{1}^{0}}$. However, in some parts of the parameter space the left sleptons can be lighter than the $\tilde{\chi}_{1}^{0}$, c.f. regions $\mathrm{C}$ in Fig. 1. In this case, the long decay chains of the type of Fig. 4(c, d, e) obviously do not occur. Instead, we have $\tilde{\chi}_{1,2}^{0} \rightarrow l^{ \pm} \tilde{l}_{L}^{\mp}$, $\nu_{l} \tilde{\nu}_{l}$ and $\tilde{\chi}_{1}^{ \pm} \rightarrow \nu \tilde{l}_{L}^{ \pm}, l^{ \pm} \tilde{\nu}_{l}$ with $l=(e, \mu)$ in addition to the decays into $\tilde{\tau}_{1}$ or $\tilde{\nu}_{\tau}$. These are followed by 3-body decays of the sleptons: $\tilde{l}_{L}^{ \pm} \rightarrow l^{ \pm} \nu_{\tau} \tilde{\nu}_{\tau}, \nu_{l} \tau^{ \pm} \tilde{\nu}_{\tau}$ and $\tilde{\nu}_{l} \rightarrow \nu_{l} \nu_{\tau} \tilde{\nu}_{\tau}, l^{ \pm} \tau^{\mp} \tilde{\nu}_{\tau}$. Some of the resulting squark decay chains are depicted in Fig. 5, A concrete example is realised by taking the parameter point of Table 1 and lowering $m_{1 / 2}$ to $m_{1 / 2}=450 \mathrm{GeV}$. The masses and branching ratios for this case, together with the slepton decay widths, are given in Table 2 ,

A special situation arises for larger $m_{H_{2}}^{2}$, as in the right panels of Figs. 1 and 2 , in which case the $\mu$ parameter becomes smaller. Consequently, the $\tilde{\chi}_{3,4}^{0}$ and $\tilde{\chi}_{2}^{ \pm}$are lighter than in the previous examples, and the $\tilde{\chi}_{1,2}^{0}$ and $\tilde{\chi}_{1}^{ \pm}$acquire sizable higgsino components. The $\tilde{q}_{L}$ then decays dominantly into $\tilde{\chi}_{4}^{0} q$ and $\tilde{\chi}_{2}^{ \pm} q^{\prime}$, while the $\tilde{q}_{R}$ decays not only into $\tilde{\chi}_{1}^{0} q$ but also into $\tilde{\chi}_{2}^{0} q$. The heavy neutralino and chargino, $\tilde{\chi}_{4}^{0}$ and $\tilde{\chi}_{2}^{ \pm}$, decay further into sleptons, gauge bosons, or $h^{0}$ with roughly comparable rates. This makes this scenario even more complicated than that of Table 1. The detection of the heavier neutralino and chargino states through their decays into sleptons has been studied in [44], and the use of hadronic neutralino/chargino decays very recently in [45]. 
(a)

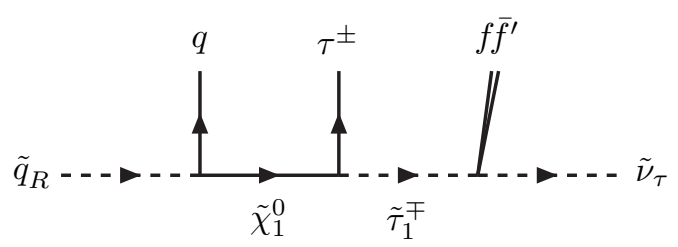

(b)

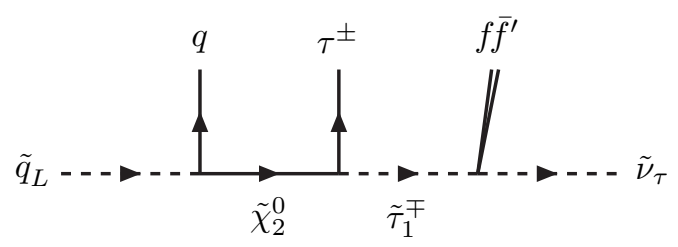

(c)

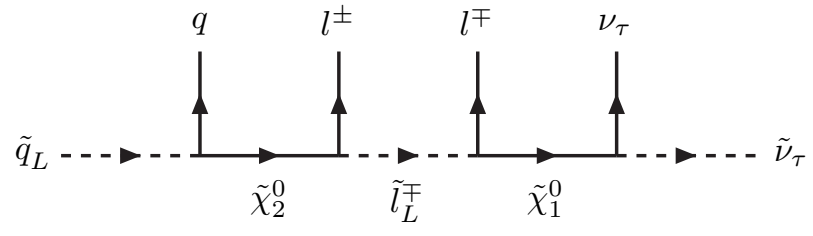

(d)

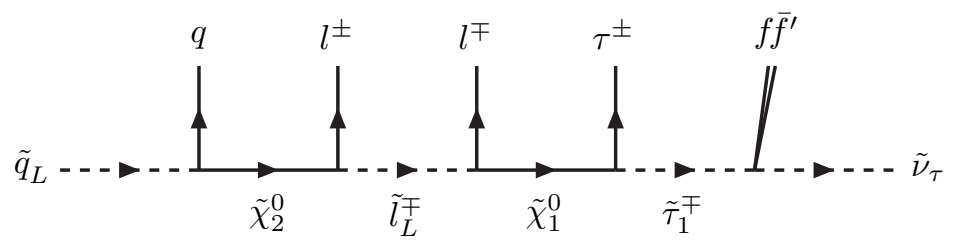

(e)

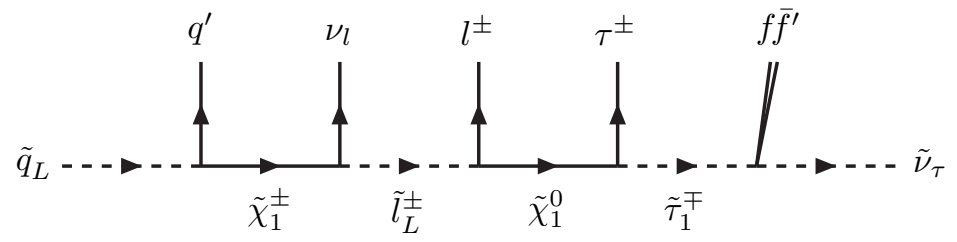

Fig. 4: Examples of squark cascade decays in gaugino mediation with a sneutrino NLSP; $l=(e, \mu)$.

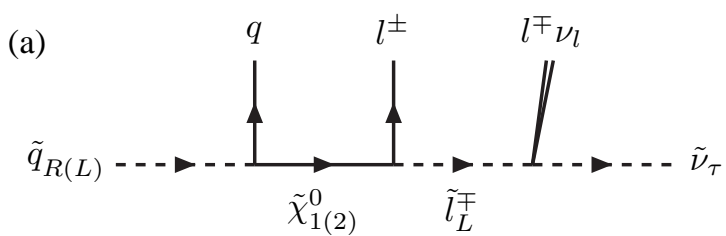

(b)

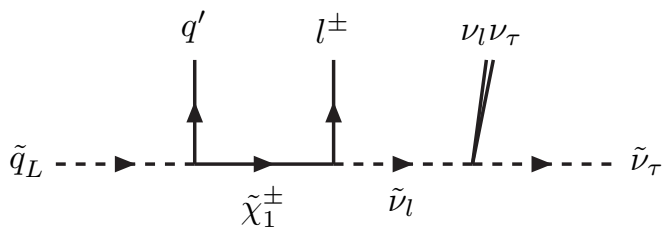

Fig. 5: Examples of squark cascade decays for the case $m_{\tilde{\chi}_{1}^{0}}>m_{\tilde{l}_{L}}$ [in addition to Fig. 4 (a,b)]. 
Table 2: Spectrum and branching ratios for $m_{1 / 2}=450 \mathrm{GeV}, \tan \beta=10, m_{H_{1}}^{2}=2.7 \mathrm{TeV}^{2}, m_{H_{2}}^{2}=0$. As the first and second generation sfermions are practically degenerate, only the first generation is given.

\begin{tabular}{|c|c|c|}
\hline Sparticle & Mass $[\mathrm{GeV}]$ & Dominant decay modes \\
\hline$g$ & 1046.1 & 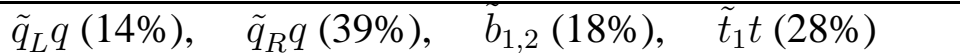 \\
\hline$\tilde{u}_{L}, \tilde{d}_{L}$ & $960.7,967.6$ & $\tilde{\chi}_{2}^{0} q(32 \%), \quad \tilde{\chi}_{1}^{ \pm} q^{\prime}(\sim 60 \%)$ \\
\hline$\tilde{u}_{R}, \tilde{d}_{R}$ & $874.9,940.8$ & $\tilde{\chi}_{1}^{0} q(99 \%)$ \\
\hline$\tilde{t}_{1}$ & 685.9 & $\tilde{\chi}_{1}^{0} t(29 \%), \quad \tilde{\chi}_{1}^{+} b(36 \%)$ \\
\hline$\tilde{\chi}_{4}^{0}$ & 560.5 & $\tilde{\chi}_{1}^{ \pm} W^{\mp}(44 \%), \quad \tilde{\chi}_{2}^{0} h(17 \%)$ \\
\hline$\tilde{\chi}_{2}^{ \pm}$ & 557.5 & $\tilde{\chi}_{2}^{0} W^{ \pm}(25 \%), \quad \tilde{\chi}_{1}^{ \pm} Z(21 \%)$ \\
\hline$\tilde{\chi}_{3}^{0}$ & 545.8 & $\tilde{\chi}_{1}^{ \pm} W^{\mp}(56 \%), \quad \tilde{\chi}_{2}^{0} Z(25 \%)$ \\
\hline$\tilde{e}_{R}$ & 411.1 & $\tilde{\chi}_{1}^{0} e(100 \%)$ \\
\hline$\tilde{\tau}_{2}$ & 391.2 & $\tilde{\chi}_{1}^{0} \tau(83 \%)$ \\
\hline$\tilde{\chi}_{1}^{ \pm}$ & 345.3 & $\tilde{e}_{L}^{ \pm} \nu_{e}(15 \%), \quad \tilde{\nu}_{e} e^{ \pm}(16 \%), \quad \tilde{\tau}_{1}^{ \pm} \nu_{\tau}(18 \%), \quad \tilde{\nu}_{\tau} \tau^{ \pm}(19 \%)$ \\
\hline$\tilde{\chi}_{2}^{0}$ & 339.5 & $\tilde{\tau}_{1}^{ \pm} \tau^{\mp}(20 \%), \quad \tilde{e}_{L}^{ \pm} e^{\mp}(16 \%), \quad \tilde{\nu}_{e} \nu_{e}(15 \%)$ \\
\hline$\tilde{\chi}_{1}^{0}$ & 181.4 & $\tilde{e}^{ \pm} e^{\mp}(8 \%), \quad \tilde{\tau}_{1}^{ \pm} \tau^{\mp}(25 \%), \quad \tilde{\nu}_{\tau} \nu_{\tau}(32 \%)$ \\
\hline$\tilde{e}_{L}$ & 142.7 & $\tilde{\nu}_{\tau} \tau \nu_{e}(\sim 100 \%), \quad \Gamma=6 \times 10^{-7} \mathrm{GeV}$ \\
\hline$\tilde{\nu}_{e}$ & 136.5 & $\tilde{\nu}_{\tau} \nu_{e} \nu_{\tau}(91 \%), \quad \tilde{\nu}_{\tau} e^{-} \tau^{+}(9 \%), \quad \Gamma=4 \times 10^{-7} \mathrm{GeV}$ \\
\hline$\tilde{\tau}_{1}$ & 106.0 & $\tilde{\nu}_{\tau} l \nu(30 \%), \quad \tilde{\nu}_{\tau} q \bar{q}^{\prime}(70 \%), \quad \Gamma=6 \times 10^{-9} \mathrm{GeV}$ \\
\hline$\tilde{\nu}_{\tau}$ & 101.3 & $\widetilde{G} \nu_{\tau}, \quad \Omega_{\tilde{\nu}}^{\text {th }} h^{2}=5.5 \times 10^{-3}$ \\
\hline
\end{tabular}

A comment is in order concerning the detectability of the soft leptons. For the parameter point of Table 1 with $m_{\tilde{\tau}_{1}}-m_{\tilde{\nu}_{\tau}} \simeq 6 \mathrm{GeV}$, for instance, the mean $p_{T}$ of the electrons and muons coming from the $\tilde{\tau}_{1} \rightarrow W^{*} \tilde{\nu}_{\tau}$ decay is $5.9 \mathrm{GeV}$ at generator level ${ }^{4}$ Requiring $p_{T}(e, \mu)>3 \mathrm{GeV}$, $5 \mathrm{GeV}$, or $10 \mathrm{GeV}$ in the offline reconstruction, about $60 \%, 40 \%$, or $17 \%$, respectively, of these leptons would pass. At first glance this may appear very challenging for LHC analyses. Notice, however, that the SUSY events can be selected by triggering on the hard jets/leptons and the $E_{T}^{\text {miss }}$, so that the detection of additional soft electrons and/or muons may well be feasible. Cuts of $p_{T}(e)>5 \mathrm{GeV}$ and $p_{T}(\mu)>3 \mathrm{GeV}$ were, for example, also used in [43] for Higgs boson search in the $H \rightarrow Z Z^{(*)} \rightarrow 4 l$ channel. The situation is of course better for larger $\tilde{\nu}_{\tau}-\tilde{\tau}_{1}$ mass difference. Taus and jets coming from the 3 -body $\tilde{\tau}_{1}$ decays will, however, hardly be observable.

At the ILC [46-48], several distinctive features of the $\tilde{\nu}_{\tau}$ NLSP scenario may be resolved with high accuracy, in particular the large mass splitting between left and right sleptons with $m_{\tilde{l}_{L}}<m_{\tilde{l}_{R}}$ (although measuring $m_{\tilde{l}_{R}}$ may require a $1 \mathrm{TeV}$ linear collider). Selectron-pair production can give $e^{+} e^{-}+E_{T}^{\text {miss }}$ or $e^{+} e^{-} \tau^{+} \tau^{-}+2\left(f \bar{f}^{\prime}\right)+E_{T}^{\text {miss }}$, and analogously for smuons and for $\tilde{\tau}_{2}$, depending on the mass orderings. (For $m_{\tilde{e}_{L}}<m_{\tilde{\chi}_{1}^{1}}$, however, pair production of $\tilde{e}_{L}$ leads to $\tau^{+} \tau^{-}+E_{T}^{\text {miss }}$ due to 3-body $\tilde{e}_{L}$ decays.) Beam polarisation, angular distributions and tunable energy can be exploited to determine the mass, chirality and spin of the sleptons.

Pair production of $\tilde{\tau}_{1}$ gives $2\left(f \bar{f}^{\prime}\right)+E_{T}^{\text {miss }}$. Since the 3-body stau decay proceeds dominantly through an off-shell $W$ boson, this results in soft jets plus missing energy in half of the cases. In addition, about $20 \%$ of the $\tilde{\tau}_{1} \tilde{\tau}_{1}^{*}$ events give jets plus a single charged lepton plus $E_{T}^{\text {miss }}$, and the remaining $\sim 10 \%$ lead to $l^{ \pm} l^{\mp}+E_{T}^{\text {miss }}$ or mixed-flavour events of, for instance, $e^{ \pm} \mu^{\mp}+E_{T}^{\text {miss }}$. On the one hand this certainly complicates the analysis, on the other hand resolving the various $l \nu_{l}$ and $q \bar{q}^{\prime}$ modes of the $\tilde{\tau}_{1}$ decay and estimating the lifetime allows one to distinguish this scenario from a stau NLSP which decays into $\tau \widetilde{G}$ [49-54], $\tau$ axino [55] or even

\footnotetext{
${ }^{4}$ We thank Are Raklev for providing the $p_{T}$ spectrum.
} 
from the case of gravitino DM with R-parity breaking [56].

Chargino production and subsequent decay into lepton and sneutrino could also provide an efficient way to measure the sneutrino mass, as in the case of neutralino LSP studied in [57].

Last but not least, pair-production of $\tilde{\chi}_{1}^{0}$ can lead to visible events from $\tilde{\chi}_{1}^{0} \rightarrow \tau^{ \pm} \tilde{\tau}_{1}^{\mp}$ decays, and in the case that $m_{\tilde{\chi}_{1}^{0}}>m_{\tilde{e}_{L}}$ also from $\tilde{\chi}_{1}^{0} \rightarrow e^{ \pm} \tilde{e}_{L}^{\mp}, \mu^{ \pm} \tilde{\mu}_{L}^{\mp}$ decays. The ISR photon spectrum may give additional information on the $\tilde{\chi}_{1}^{0}$ and $\tilde{\nu}_{\tau}$ masses.

\section{Conclusions}

We have considered the case of gravitino LSP and dark matter with a sneutrino NLSP in the scenario of gaugino-mediated supersymmetry breaking. We find viable regions of the parameter space, where the primordial sneutrino abundance satisfies the BBN constraints. A general feature of this scenario is a small mass splitting between the $\tilde{\tau}_{1} \sim \tilde{\tau}_{L}$ and the $\tilde{\nu}_{\tau}$, leading to 3-body $\tilde{\tau}_{1}$ decays into $f \bar{f}^{\prime} \tilde{\nu}_{\tau}$, dominantly mediated by a virtual $W$. This can significantly influence the SUSY collider signatures. We have discussed these signatures depending on the mass ordering of $\tilde{\chi}_{1,2}^{0}, \tilde{\tau}_{1}$ and $\tilde{e}_{L}$. In particular, if $m_{\tilde{\chi}_{1}^{0}}>m_{\tilde{\tau}_{1}}+m_{\tau}$ (and/or $\left.m_{\tilde{e}_{L}}\right)$, the lightest neutralino can have visible decays into a charged lepton and slepton. Moreover, for $m_{\tilde{\chi}_{1}^{0}}>m_{\tilde{e}_{L}}$, also selectrons and smuons will only have 3-body decays into the $\tilde{\nu}_{\tau}$. These 3-body decays do, however, not lead to displaced vertices unless the spectrum is quasi-degenerate.

In general this scenario predicts more soft leptons or jets in the final states and longer decay chains. Detailed simulation studies will be necessary to assess the experimental precisions achievable at the LHC or ILC in the scenarios discussed here. This is, however, beyond the scope of this letter.

\section{Acknowledgements}

We would like to thank Wolfgang Adam, Ben Allanach, Jörn Kersten, Giacomo Polesello, Alexander Pukhov and Kai Schmidt-Hoberg, for useful discussions.

S.K. is supported by an APART (Austrian Programme for Advanced Research and Technology) grant of the Austrian Academy of Sciences. L.C. acknowledges the support of the "Impuls- und Vernetzungsfonds" of the Helmholtz Association, contract number VH-NG-006.

\section{References}

[1] M. Bolz, A. Brandenburg and W. Buchmuller, Nucl. Phys. B606, 518 (2001), [hep-ph/0012052].

[2] J. Pradler and F. D. Steffen, Phys. Rev. D75, 023509 (2007), [hep-ph/0608344].

[3] J. Hamann, S. Hannestad, M. S. Sloth and Y. Y. Y. Wong, Phys. Rev. D75, 023522 (2007), [astro-ph/0611582].

[4] J. L. Feng, A. Rajaraman and F. Takayama, Phys. Rev. Lett. 91, 011302 (2003), [hep-ph/0302215].

[5] M. Kawasaki, K. Kohri and T. Moroi, Phys. Rev. D71, 083502 (2005), [astro-ph/0408426].

[6] F. D. Steffen, JCAP 0609, 001 (2006), [hep-ph/0605306].

[7] M. Pospelov, hep-ph/0605215. 
[8] K. Kohri and F. Takayama, hep-ph/0605243.

[9] M. Kaplinghat and A. Rajaraman, Phys. Rev. D74, 103004 (2006), [astro-ph/0606209].

[10] K. Hamaguchi, T. Hatsuda, M. Kamimura, Y. Kino and T. T. Yanagida, hep-ph/0702274.

[11] J. Pradler and F. D. Steffen, Phys. Lett. B 648224 (2007), [arXiv:hep-ph/0612291].

[12] J. R. Ellis, K. A. Olive, Y. Santoso and V. C. Spanos, Phys. Lett. B588, 7 (2004), [hep-ph/0312262].

[13] J. L. Feng, S. Su and F. Takayama, Phys. Rev. D70, 075019 (2004), [hep-ph/0404231].

[14] L. Roszkowski, R. Ruiz de Austri and K.-Y. Choi, JHEP 08, 080 (2005), [hep-ph/0408227].

[15] D. G. Cerdeno, K.-Y. Choi, K. Jedamzik, L. Roszkowski and R. Ruiz de Austri, JCAP 0606, 005 (2006), [hep-ph/0509275].

[16] K. Jedamzik, Phys. Rev. D74, 103509 (2006), [hep-ph/0604251].

[17] R. H. Cyburt, J. Ellis, B. D. Fields, K. A. Olive and V. C. Spanos, JCAP 0611, 014 (2006), [astro-ph/0608562].

[18] J. L. Diaz-Cruz, J. Ellis, K. A. Olive and Y. Santoso, hep-ph/0701229.

[19] J. L. Feng, S.-f. Su and F. Takayama, Phys. Rev. D70, 063514 (2004), [hep-ph/0404198].

[20] T. Kanzaki, M. Kawasaki, K. Kohri and T. Moroi, Phys. Rev. D75, 025011 (2007), [hep-ph/0609246].

[21] J. R. Ellis, T. Falk, K. A. Olive and Y. Santoso, Nucl. Phys. B652, 259 (2003), [hep-ph/0210205].

[22] D. E. Kaplan, G. D. Kribs and M. Schmaltz, Phys. Rev. D62, 035010 (2000), [hep-ph/9911293].

[23] Z. Chacko, M. A. Luty, A. E. Nelson and E. Ponton, JHEP 01, 003 (2000), [hep-ph/9911323].

[24] J. R. Ellis, C. Kounnas and D. V. Nanopoulos, Nucl. Phys. B247, 373 (1984).

[25] K. Inoue, M. Kawasaki, M. Yamaguchi and T. Yanagida, Phys. Rev. D45, 328 (1992).

[26] W. Buchmuller, J. Kersten and K. Schmidt-Hoberg, JHEP 02, 069 (2006), [hep-ph/0512152].

[27] J. L. Evans, D. E. Morrissey and J. D. Wells, hep-ph/0611185.

[28] W. Buchmuller, L. Covi, J. Kersten and K. Schmidt-Hoberg, JCAP 0611, 007 (2006), [hep-ph/0609142].

[29] B. C. Allanach, Comput. Phys. Commun. 143, 305 (2002), [hep-ph/0104145], http://projects.hepforge.org/softsusy/. 
[30] G. Belanger, F. Boudjema, A. Pukhov and A. Semenov, Comput. Phys. Commun. 149, 103 (2002), [hep-ph/0112278].

[31] G. Belanger, F. Boudjema, A. Pukhov and A. Semenov, Comput. Phys. Commun. 174, 577 (2006), [hep-ph/0405253].

[32] G. Belanger, F. Boudjema, A. Pukhov and A. Semenov, hep-ph/0607059, http://wwwlapp.in2p3.fr/lapth/micromegas/.

[33] T. Asaka, K. Hamaguchi and K. Suzuki, Phys. Lett. B490, 136 (2000), [hep-ph/0005136].

[34] T. Falk, K. A. Olive and M. Srednicki, Phys. Lett. B339, 248 (1994), [hep-ph/9409270].

[35] M. Muhlleitner, A. Djouadi and Y. Mambrini, Comput. Phys. Commun. 168, 46 (2005), [hep-ph/0311167].

[36] A. Pukhov, hep-ph/0412191.

[37] I. Hinchliffe, F. E. Paige, M. D. Shapiro, J. Soderqvist and W. Yao, Phys. Rev. D55, 5520 (1997), [hep-ph/9610544].

[38] H. Bachacou, I. Hinchliffe and F. E. Paige, Phys. Rev. D62, 015009 (2000), [hep-ph/9907518].

[39] B. C. Allanach, C. G. Lester, M. A. Parker and B. R. Webber, JHEP 09, 004 (2000), [hep-ph/0007009].

[40] C. G. Lester, CERN-THESIS-2004-003.

[41] D. J. Miller, P. Osland and A. R. Raklev, JHEP 03, 034 (2006), [hep-ph/0510356].

[42] ATLAS Collaboration, ATLAS Detector and Physics Performance: Technical Design Report, vol. 2, 1999, ATLAS-TDR-15, CERN-LHCC-99-15.

[43] CMS Collaboration, CMS Physics: Technical Design Report, Volume 2: Physics Performance, 2006, CMS-TDR-8.2, CERN-LHCC-2006-021.

[44] G. Polesello, J. Phys. G30, 1185 (2004).

[45] J. M. Butterworth, J. R. Ellis and A. R. Raklev, hep-ph/0702150.

[46] ECFA/DESY LC Physics Working Group, J. A. Aguilar-Saavedra et al., TESLA Technical Design Report. Part III: Physics at an $e^{+} e^{-}$Linear Collider, 2001, hep-ph/0106315.

[47] American Linear Collider Working Group, T. Abe et al., Linear collider physics resource bookfor Snowmass 2001. 2: Higgs and supersymmetry studies, 2001, hep-ex/0106056.

[48] ACFA Linear Collider Working Group, K. Abe et al., Particle physics experiments at JLC, 2001, hep-ph/0109166.

[49] W. Buchmuller, K. Hamaguchi, M. Ratz and T. Yanagida, Phys. Lett. B588, 90 (2004), [hep-ph/0402179]. 
[50] K. Hamaguchi, Y. Kuno, T. Nakaya and M. M. Nojiri, Phys. Rev. D70, 115007 (2004), [hep-ph/0409248].

[51] J. L. Feng and B. T. Smith, Phys. Rev. D71, 015004 (2005), [hep-ph/0409278].

[52] H. U. Martyn, Eur. Phys. J. C48, 15 (2006), [hep-ph/0605257].

[53] J. R. Ellis, A. R. Raklev and O. K. Oye, JHEP 10, 061 (2006), [hep-ph/0607261].

[54] K. Hamaguchi, M. M. Nojiri and A. de Roeck, hep-ph/0612060.

[55] A. Brandenburg, L. Covi, K. Hamaguchi, L. Roszkowski and F. D. Steffen, Phys. Lett. B617, 99 (2005), [hep-ph/0501287].

[56] W. Buchmuller, L. Covi, K. Hamaguchi, A. Ibarra and T. Yanagida, hep-ph/0702184.

[57] A. Freitas, W. Porod and P. M. Zerwas, Phys. Rev. D 72 (2005) 115002 [arXiv:hep-ph/0509056]. 